\title{
Precise nuclear matter densities from heavy-ion collisions
}

\author{
M. A. G. Alvarez, ${ }^{1}$ E. S. Rossi, Jr., ${ }^{1}$ C. P. Silva, ${ }^{1}$ L. R. Gasques, ${ }^{1}$ L. C. Chamon, ${ }^{1}$ D. Pereira, ${ }^{1}$ M. N. Rao, ${ }^{1}$ B. V. Carlson, ${ }^{2}$ \\ C. De Conti, ${ }^{2}$ R. M. Anjos, ${ }^{3}$ P. R. S. Gomes, ${ }^{3}$ J. Lubian, ${ }^{3}$ S. Kailas, ${ }^{4}$ A. Chatterjee, ${ }^{4}$ and P. Singh ${ }^{4}$ \\ ${ }^{1}$ Laboratório Pelletron, Instituto de Física da Universidade de São Paulo, 05315-970 São Paulo, SP, Brazil \\ ${ }^{2}$ Departamento de Física, Instituto Tecnológico de Aeronáutica, Centro Técnico Aeroespacial, São José dos Campos, SP, Brazil \\ ${ }^{3}$ Instituto de Física, Universidade Federal Fluminense, Av. Litoranea, Niterói 24210-340, RJ, Brazil \\ ${ }^{4}$ Nuclear Physics Division, Bhabha Atomic Research Centre, Bombay 400 085, India
}

(Received 3 April 2001; published 30 November 2001)

\begin{abstract}
An unfolding method is proposed to extract ground-state nuclear matter densities from heavy-ion elastic scattering data analyses at low (sub-barrier) and intermediate energies. The consistency of the results is fully checked. The method should be of value in determining densities for exotic nuclei.
\end{abstract}

DOI: 10.1103/PhysRevC.65.014602

A long-standing question of nuclear structure concerns the determination of heavy-ion neutron densities, which are far from being as well known as the proton densities that have been extracted from electron scattering experiments. It is worth mentioning the importance of the determination of nuclear densities to distinguish among different nuclear structure theoretical approaches. Several probes (pion, proton, alpha, etc.) have been used in order to determine nuclear matter densities, with different sorts of limitations [1]. For instance, the use of the strong interacting probes $\pi^{+}$and $\pi^{-}$ is usually accompanied by the need to "calibrate" the method, which means that only average radii and differences in densities are the most reliable results. In a more fundamental philosophy, the possibility of extracting information on nuclear distributions from heavy-ion elastic scattering is a question of using the folding model for the interaction, including all the important effects from first principles and avoiding the use of adjustable parameters as much as possible.

In the present work, a method of determining matter densities from heavy-ion elastic scattering data at sub-barrier and intermediate energies is proposed. It is based on the parameter-free nonlocal energy-independent bare potential (NLM3Y potential), recently developed [2-5] for the real part of the nucleus-nucleus interaction. The NLM3Y potential has been tested for several systems $[3,4]$ and gives excellent reproductions of measured elastic and inelastic cross sections in a large energy range, particularly at intermediate energies where the refractive elastic data are very sensitive to the real part of the interaction [6]. The model (for details see [3]) takes into account the Pauli nonlocality involving the exchange of nucleons between the target and the projectile. The energy-independent real part of the interaction is given by

$$
V\left(\vec{R}, \vec{R}^{\prime}\right)=V_{N L}\left(\frac{R+R^{\prime}}{2}\right) \frac{1}{\pi^{3 / 2} b^{3}} e^{-\left(\left|\vec{R}-\vec{R}^{\prime}\right| / b\right)^{2}}
$$

where $b=b_{0} m_{0} / \mu$ is the range of the Pauli nonlocality, $b_{0}$ $=0.85 \mathrm{fm}, m_{0}$ and $\mu$ are the nucleon mass and the reduced mass of the system, respectively. The nonlocal interaction is connected to the usual folding potential [7] through
PACS number(s): 25.70.Bc, 21.10.Gv, 21.10.Ft, 24.10.Ht

$$
V_{N L}(R)=\int \rho_{1}\left(r_{1}\right) v\left(\vec{R}-\vec{r}_{1}+\vec{r}_{2}\right) \rho_{2}\left(r_{2}\right) d \vec{r}_{1} d \vec{r}_{2},
$$

where $\rho_{1}\left(r_{1}\right)$ and $\rho_{2}\left(r_{2}\right)$ are the ground-state nuclear densities of the colliding partners, and $v(\vec{r})$ is the M3Y effective nucleon-nucleon interaction. The corresponding energydependent local equivalent potential is given by [3]

$$
V_{L E}(R ; E)=\frac{1-\sqrt{1-4 \gamma V_{N L}(R) e^{-\gamma\left[E-V_{C}(R)\right]}}}{2 \gamma},
$$

with $\gamma=\mu b^{2} / 2 \hbar^{2}$. We mention in passing that other approaches for the finite range exchange term (for example see $[8-10])$ are more complicated to calculate and therefore less suitable for extensive studies of nuclear densities.

Within the model above, the central idea of the method proposed is to extract ground-state nuclear distributions from elastic scattering data analyses, with the densities as the result of best fits in an unfolding procedure involving expressions (2) and (3). The data analyses at intermediate energies give information about the total (neutron + proton) distributions in a region close to the root-mean-square radius $\left(r_{\mathrm{rms}}\right)$, while at sub-barrier energies the surface is the region sensitive to the data fits.

To characterize the absorption from reaction channels, at intermediate energies we have used an imaginary potential based on the Lax-type interaction [11]:

$$
W(R ; E)=-\frac{E}{k_{N}} \sigma_{T}^{N N}(E) \int \rho_{1}(|\vec{R}-\vec{r}|) \rho_{2}(r) d \vec{r},
$$

where $\sigma_{T}^{N N}(E)$ is the average nucleon-nucleon total cross section with Pauli blocking. For the sub-barrier case, we have selected elastic scattering experimental angular distributions at energies sufficiently below the Coulomb barrier, that couplings to reaction channels are very small. In this case, we have used an inner imaginary potential with WoodsSaxon shape, which takes into account the small internal absorption from barrier penetration. The values adopted for the parameters of this potential result in small strengths at the surface region. This procedure must be used in the subbarrier data analyses due to the small cross sections of peripherical reaction channels. No sensitivity in the cross sec- 


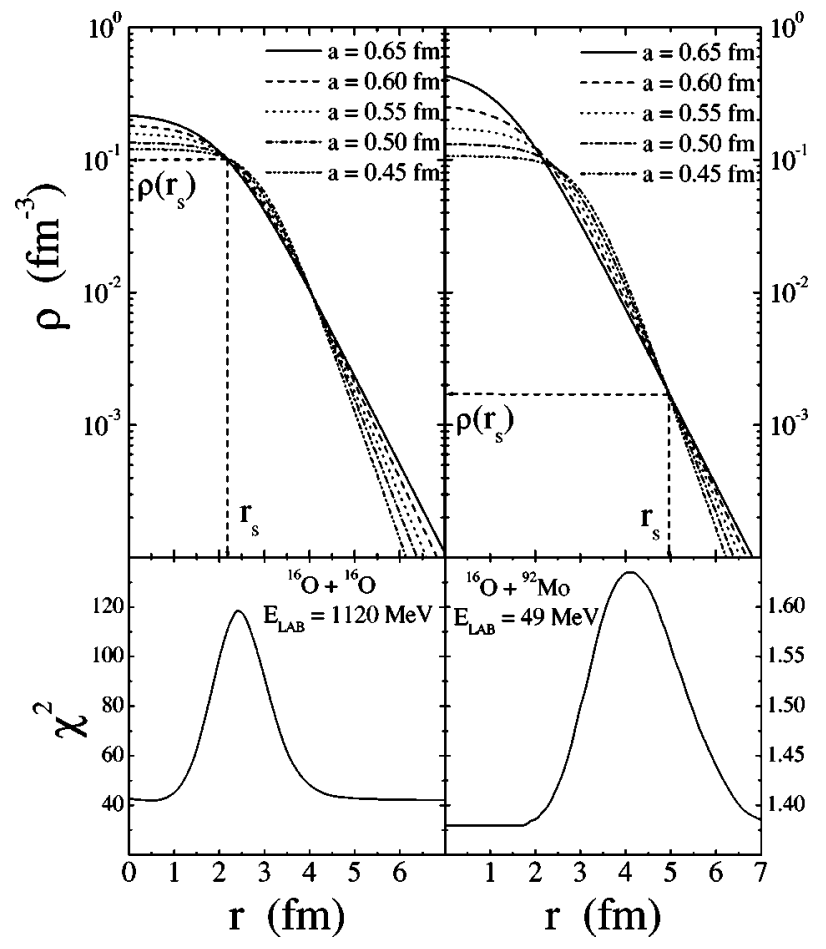

FIG. 1. Top: Examples of the determination of the sensitivity radii, $r_{s}$, and the corresponding experimental values for the ${ }^{16} \mathrm{O}$ nuclear matter density, $\rho\left(r_{s}\right)$, using two-parameter Fermi distributions which give equivalent elastic scattering data fits for angular distributions of the ${ }^{16} \mathrm{O}+{ }^{16} \mathrm{O}\left(E_{l a b}=1120 \mathrm{MeV}\right)$ and ${ }^{16} \mathrm{O}+{ }^{92} \mathrm{Mo}$ $\left(E_{l a b}=49 \mathrm{MeV}\right)$ systems. Bottom: The sensitivity regions for the ${ }^{16} \mathrm{O}$ nuclear matter density characterized by notch tests.

tion predictions has been detected related to depth variations of this absorptive potential. We point out that the polarization potential that arises from reaction channel couplings (Feshbach nonlocality) has been estimated [12-14] through extensive coupled channel calculations for the sub-barrier data set, and represents less than $10 \%$ in comparison with the bare (folding) interaction.

We have chosen ${ }^{16} \mathrm{O}$ as a test case, due to the extensive experimental and theoretical information available about this nucleus, and, as discussed in Ref. [12], because different approaches give quite different results for the ${ }^{16} \mathrm{O}$ nuclear density, particularly at the surface region. In the analyses, we have assumed a two-parameter Fermi model (2PF) for the ${ }^{16} \mathrm{O}$ density, with diffuseness $(a)$ and radius $\left(R_{0}\right)$ searched for the best data fits, and with the $\rho_{0}$ parameter determined by the normalization condition

$$
4 \pi \int_{0}^{\infty} \frac{\rho_{0}}{1+\exp \left(\frac{r-R_{0}}{a}\right)} r^{2} d r=16 .
$$

In Fig. 1 is presented, as an illustration of our method, the determination of the total (neutron + proton) density for the ${ }^{16} \mathrm{O}$ nucleus at the $r_{\text {rms }}$ radius and surface regions, by using elastic scattering data analyses at intermediate and subbarrier energies, respectively. For each angular distribution, we have found a family of densities which give equivalent

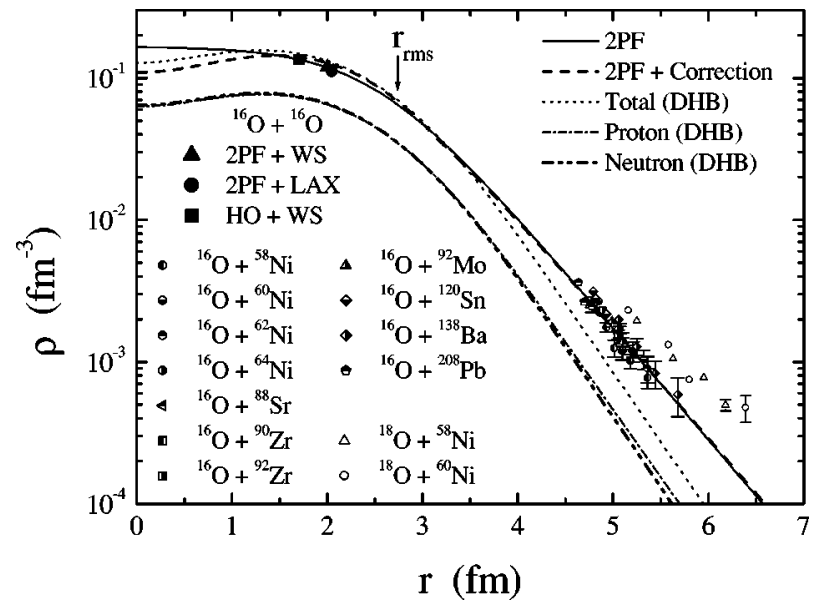

FIG. 2. Experimental nuclear density values for the ${ }^{16} \mathrm{O}$ (semiclosed symbols) and ${ }^{18} \mathrm{O}$ (open symbols) nuclei, as obtained from sub-barrier elastic scattering data analyses for different systems and bombarding energies. The closed symbols represent density values $\left({ }^{16} \mathrm{O}\right)$ from intermediate energy data analyses $\left({ }^{16} \mathrm{O}+{ }^{16} \mathrm{O}, E_{l a b}\right.$ $=1120 \mathrm{MeV}$ ), using different models for the shape of the ${ }^{16} \mathrm{O}$ density (2PF or HO) and for the imaginary potential (WS or Lax). The lines correspond to theoretical Dirac-Hartree-Bogoliubov (DHB) calculations for the ${ }^{16} \mathrm{O}$ nucleus, and a two-parameter Fermi distribution (2PF) with or without a damped oscillatory correction.

data fits. These densities cross (Fig. 1 top) at a particular radius $r_{s}$, hereafter referred to as the sensitivity radius. Similar behavior has been observed in the determination of bare potentials from sub-barrier data analyses [12-15], but in that case only one crossing was detected for each angular distribution. In the density case, two crossings are observed (Fig. 1) due to the particular shape and normalization condition imposed on the nuclear density. Thus, the determination of the sensitivity radius is also accompanied by a notch test (Fig. 1 bottom), in which a spline with Gaussian shape is included in the ${ }^{16} \mathrm{O}$ density, and the variation of the chisquare is studied as a function of the position of this perturbation. The notch test guarantees that $r_{s}$ is in a density region important for the data fit, and does not arise from spurious crossing. Since the data fits depend only on the density in a small range of nuclear radii, the determination of the sensitivity radius and corresponding density value is rather independent of the shape assumed for the nuclear distribution (2PF, harmonic oscillator - see example in Fig. 2). For the ${ }^{16} \mathrm{O}+{ }^{16} \mathrm{O}$ system at the energy of $1120 \mathrm{MeV}$, besides the Lax interaction we have also used a three free parameter imaginary potential, with Woods-Saxon shape, with the aim of evaluating any possible change in the sensitivity radius. The $r_{s}$ and corresponding density values obtained in this case are quite similar to those from the Lax interaction (see Fig. 2).

The ${ }^{16} \mathrm{O}$ experimental density values at the sensitivity radii obtained from heavy-ion data analyses are shown in Fig. 2. For the sub-barrier energies, the elastic scattering data [12-15] are from 40 angular distributions of 11 systems like ${ }^{16} \mathrm{O}+A$, where $A$ is a magic or semimagic target nucleus with mass number ranging from $58(\mathrm{Ni})$ to $208(\mathrm{~Pb})$. In the data analyses, we have used Hartree-Fock, Dirac-Hartree- 
Bogoliubov, and shell-model densities for the target nuclei (see Refs. [12-16]). In this sub-barrier region the position of the sensitivity radius is energy-dependent, with variation connected to the classical turning point of the effective potential. This fact allows us to characterize the ${ }^{16} \mathrm{O}$ nuclear distribution (semiclosed symbols in Fig. 2) in a large and superficial region. The data (from Ref. [17]) analyses at $E_{l a b}=1120 \mathrm{MeV}$ for the ${ }^{16} \mathrm{O}+{ }^{16} \mathrm{O}$ system have provided information of the ${ }^{16} \mathrm{O}$ density in a much inner region (closed symbols in Fig. 2). A theoretical prediction for the ${ }^{16} \mathrm{O}$ density derived from the Dirac-Hartree-Bogoliubov (DHB) model [18] using NL3 potential parameters [19] is also shown in Fig. 2. In the surface region, the experimental ${ }^{16} \mathrm{O}$ density is much greater than the theoretical prediction. An analysis of the single-particle levels of the theoretical calculation shows, as one might expect, that the falloff of the density in the surface region is determined by the least bound levels. Although the NL3 parameter set was adjusted to reproduce binding energies and charge and neutron radii across the periodic table, it did not take into account single-particle properties, which suggests a direction for future improvements in such a parameter set.

For the purpose of comparison and demonstration of the sensitivity of the method, we have also shown in Fig. 2 the experimental density values for the ${ }^{18} \mathrm{O}$ nucleus (open symbols) obtained with the same method through optical model analyses of sub-barrier elastic scattering data for the ${ }^{18} \mathrm{O}$ $+{ }^{58,60} \mathrm{Ni}$ systems. As theoretically expected [20] and clearly demonstrated by our results, the two extra neutrons of the ${ }^{18} \mathrm{O}\left(2 s_{1 / 2}, 1 d_{3 / 2}\right.$, and $1 d_{5 / 2}$ orbitals $)$ increase the ${ }^{18} \mathrm{O}$ density at the surface region in comparison to that for the ${ }^{16} \mathrm{O}$ nucleus.

In our method, the experimental density values have been extracted based on very fundamental grounds. The parameter-free real part of the interaction contains as basic inputs just the well-known M3Y effective nucleon-nucleon interaction and the model for the Pauli nonlocality, which has been tested extensively [2-5]. Also the imaginary part of the interaction has been based on general assumptions: the lack of superficial absorption at sub-barrier energies and the parameter-free Lax-type interaction (for the ${ }^{16} \mathrm{O}+{ }^{16} \mathrm{O}$ system at $E_{l a b}=1120 \mathrm{MeV}$ ), which is known to be appropriate for high energies [11]. The adjustable parameters of the method ( $R_{0}$ and $a$ ) are connected only with the quantity to be determined: the projectile nuclear density, and the results obtained are rather insensitive to the (realistic) shape assumed for the distribution. We mention that other experimental data for the ${ }^{16} \mathrm{O}$ density in the region $2 \leqslant r \leqslant 4.5 \mathrm{fm}$ could be found through the analyses of other angular distributions in energies above the barrier, but in this case the imaginary potential must have adjustable parameters and the reliability of the results for the density should be studied very carefully [21]. Thus, we consider the theoretical densities for the target nuclei (in the sub-barrier data analysis) as the only assumption of our method that needs to be checked. The good agreement among the results for the ${ }^{16} \mathrm{O}$ density obtained using different target nuclei indicate that any possible deviation in such theoretical calculations would be systematic.

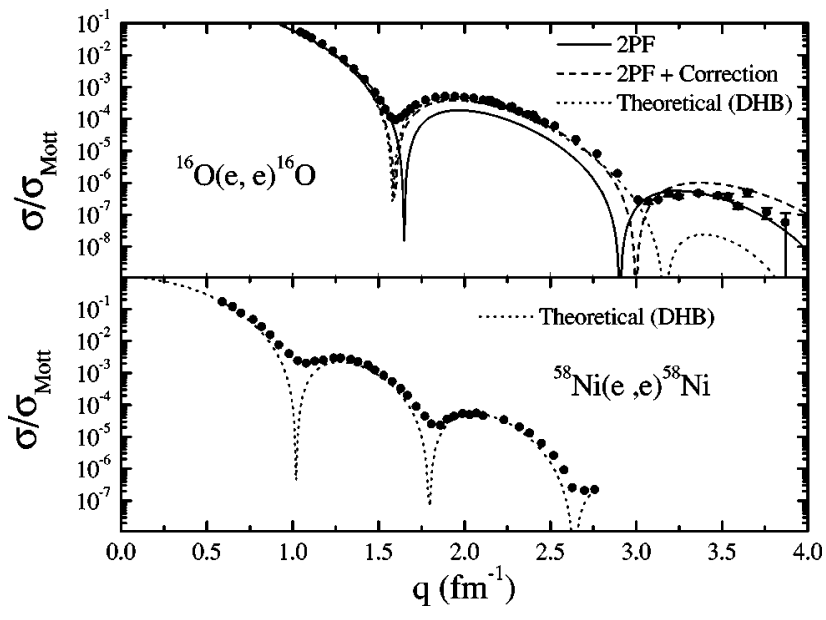

FIG. 3. Experimental electron scattering cross sections for the (top) ${ }^{16} \mathrm{O}$ and (bottom) ${ }^{58} \mathrm{Ni}$ nuclei as a function of the momentum transferred. The dotted lines in the figure are theoretical predictions using charge distributions from Dirac-Hartree-Bogoliubov (DHB) calculations in the plane-wave Born approximation. The other lines (top) are the results for charge distributions derived from experimental nuclear matter densities, using 2PF shapes (as shown in Fig. 2) with (dashed line) or without (solid line) a damped oscillatory correction.

Thus, as a test of the consistency of the assumed hypothesis of the method, we compare in Fig. 3 the data (from Refs. $[22,23])$ with predictions for electron scattering cross sections. We have used charge distributions obtained by folding the proton density of the nucleus with the intrinsic charge distribution of the proton. For the doubly-magic ${ }^{16} \mathrm{O}$ nucleus, the proton density is quite close to one-half of the total density (see the theoretical neutron and proton distributions in Fig. 2). The electron scattering cross sections have been calculated in the plane-wave Born approximation, which, for light nuclei such as ${ }^{16} \mathrm{O}$, should produce cross sections close to the exact phase-shift method, except for momentum transferred near a minimum of diffraction.

Considering a best fit $2 \mathrm{PF}$ distribution $\left(R_{0}=2.49 \mathrm{fm}\right.$ and $a=0.55 \mathrm{fm}$ - solid line in Fig. 2) to describe the ${ }^{16} \mathrm{O}$ density, a reasonable description of the electron scattering (solid line in Fig. 3 top) is obtained, with some discrepancies in the momentum transferred region $1.5 \leqslant q \leqslant 3.0 \mathrm{fm}^{-1}$. Based on the theoretical calculations for the ${ }^{16} \mathrm{O}$ density (see Fig. 2), such discrepancies are understood considering the decreasing contribution of the $1 p_{3 / 2}$ and $1 p_{1 / 2}$ components for the nuclear density in the inner radius region. We have taken this into account by adding a damped oscillatory function to the $2 \mathrm{PF}$ distribution ( $2 \mathrm{PF}+$ correction in Fig. 2), resulting in a better overall description of the electron cross section (dashed line in Fig. 3 top). As reported earlier [22], a similar procedure has been adopted to improve ${ }^{16} \mathrm{O}$ electron scattering data fits that have been obtained by using fenomenological charge densities. These fits [22] have precision comparable to those of the present work. We point out that the disagreement between predicted and measured cross sections near the minima of diffraction $\left(q \approx 1.5 \mathrm{fm}^{-1}\right.$ and $q$ $\approx 3 \mathrm{fm}^{-1}$ ) is due to the use of the Born approximation in the cross section calculations. Thus, for the first time, it is 
possible to describe electron scattering cross sections from an experimental ${ }^{16} \mathrm{O}$ nuclear density obtained through heavyion elastic scattering data analyses. The theoretical DiracHartree-Bogoliubov (DHB) charge distribution predicts electron scattering cross sections which are in disagreement with the data for large $q$ values (see Fig. 3 top). We point out that, as a further test of the consistency of the assumed hypothesis of the method, the theoretical DHB distributions for the target nuclei used in this work predict electron scattering cross sections in agreement with the data (as illustrated in Fig. 3 bottom for the ${ }^{58} \mathrm{Ni}$ ).

In conclusion, using the progress reached in the last 20 years to describe heavy-ion elastic scattering, it is possible to determine ground-state nuclear matter densities. The method presented in this work should be of value in studying densities of exotic nuclei, particularly at the surface region through sub-barrier heavy-ion elastic scattering. We point out that the difference between densities of exotic and neighboring stable nuclei is much emphasized in the surface region. This seems to be borne out by preliminary results [24] for the ${ }^{6} \mathrm{He}$ nucleus in comparison to ${ }^{4} \mathrm{He}$.

This work was partially supported by Financiadora de Estudos e Projetos (FINEP), Fundação de Amparo à Pesquisa do Estado de São Paulo (FAPESP), Fundação de Amparo à Pesquisa do Estado do Rio de Janeiro (FAPERJ), and Conselho Nacional de Desenvolvimento Científico e Tecnológico (CNPq).
[1] C.J. Batty, E. Friedman, H.J. Gils, and H. Hebel, Adv. Nucl. Phys. 19, 1 (1989).

[2] M.A.C. Ribeiro, L.C. Chamon, D. Pereira, M.S. Hussein, and D. Galetti, Phys. Rev. Lett. 78, 3270 (1997).

[3] L.C. Chamon, D. Pereira, M.S. Hussein, M.A.C. Ribeiro, and D. Galetti, Phys. Rev. Lett. 79, 5218 (1997).

[4] L.C. Chamon, D. Pereira, and M.S. Hussein, Phys. Rev. C 58, 576 (1998).

[5] D. Galetti, S.S. Mizrahi, L.C. Chamon, D. Pereira, M.S. Hussein, and M.A.C. Ribeiro, Phys. Rev. C 58, 1627 (1998).

[6] M.E. Brandan and G.R. Satchler, Phys. Rep. 285, 143 (1997).

[7] G.R. Satchler and W.G. Love, Phys. Rep. 55, 183 (1979).

[8] D.T. Khoa, Nucl. Phys. A484, 376 (1988).

[9] D.T. Khoa, W. von Oertzen, and A.A. Ogloblin, Nucl. Phys. A602, 98 (1996).

[10] D.T. Khoa, G.R. Satchler, and W. von Oertzen, Phys. Rev. C 56, 954 (1997).

[11] See, e.g., M.S. Hussein, R.A. Rego, and C.A. Bertulani, Phys. Rep. 201, 279 (1991).

[12] M.A.G. Alvarez, L.C. Chamon, D. Pereira, E.S. Rossi, Jr., C.P. Silva, L.R. Gasques, H. Dias, and M.O. Roos, Nucl. Phys. A656, 187 (1999).

[13] L.C. Chamon, D. Pereira, E.S. Rossi, Jr., C.P. Silva, R. Licht- enthaler Filho, and L.C. Gomes, Nucl. Phys. A582, 305 (1995).

[14] L.C. Chamon, D. Pereira, E.S. Rossi, Jr., C.P. Silva, H. Dias, L. Losano, and C.A.P. Ceneviva, Nucl. Phys. A597, 253 (1996).

[15] C.P. Silva, M.A.G. Alvarez, L.C. Chamon, D. Pereira, M.N. Rao, E.S. Rossi, Jr., L.R. Gasques, M.A.E. Santo, R.M. Anjos, J. Lubian, P.R.S. Gomes, C. Muri, B.V. Carlson, S. Kailas, A. Chatterjee, P. Singh, A. Shrivastava, K. Mahata, and S. Santra, Nucl. Phys. A679, 287 (2001).

[16] J.W. Negele, Phys. Rev. C 4, 1260 (1970).

[17] F. Nuoffer, G. Bartnitzky, H. Clement, A. Blazevic, H.G. Bohlen, B. Gebauer, W. von Oertzen, M. Wilpert, T. Wilpert, A. Lepine-Szily, W. Mittig, A.N. Ostrowski, and P. RousselChomaz, Nuovo Cimento A 111, 971 (1998).

[18] B.V. Carlson and D. Hirata, Phys. Rev. C 62, 054310 (2000).

[19] G.A. Lalazissis, J. Konig, and P. Ring, Phys. Rev. C 55, 540 (1997).

[20] H. Miyake and A. Mizukami, Phys. Rev. C 41, 329 (1990).

[21] M. A. G. Alvarez et al. (work in progress).

[22] I. Sick and J.S. McCarthy, Nucl. Phys. A150, 631 (1970).

[23] J.R. Ficenec, W.P. Trower, J. Heisenberg, and I. Sick, Phys. Lett. 32B, 460 (1970).

[24] L. R. Gasques et al. (work in progress). 